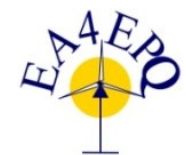

International Conference on Renewable Energies and Power Quality (ICREPQ'16)

Madrid (Spain), $4^{\text {th }}$ to $6^{\text {th }}$ May, 2016

Reneurable Energy and Power Quality. Tournal(RE\&PQJ)

ISSN 2172-038 X, No.14 May 2016

\title{
Optical high voltage breakdown prediction using thermal lensing effect in transformer oil
}

\author{
R. Struebig and I. Glesk \\ Department of Electronic and Electrical Engineering \\ University of Strathclyde \\ 204 George street, Glasgow G11XW, Scotland, UK
}

Phone/Fax number: +441415482529/+44-141-552-4968, e-mail: robinstruebig@web.de, ivan.glesk@strath.ac.uk

\begin{abstract}
We describe an optical system to monitor microscopic pre-breakdown events in liquid insulation. The system has successfully demonstrated its ability to predict high voltage breakdown in transformer oil. A simple theory based on a thermal lens build up between electrodes as a result of applied voltage is presented to explain the system operation and obtained measured results.
\end{abstract}

\section{Key words}

Breakdown prediction, Liquid insulator, High voltage, Oil movement, Laser measurements.

\section{Introduction}

Expensive assets such as power transformers are vital parts of modern power transmission networks. The value of network components lies not only in their price but also in the loss of profit if they become in operational due to the damage.

Therefore, it is crucial to develop new and effective methods for their condition monitoring and the ability to predict high voltage (HV) breakdowns. This will allow network providers to improve the reliability and reduce breakdown costs through appropriate maintenance [1].

The aim of this paper is to demonstrate a simple optical system for monitoring and electrical breakdown prediction in liquid insulation.

\section{Pre breakdown events in liquid insulation}

Stress induced oil movements by a strong electric field $\mathbf{E}$ where recorded in earlier research [2]. The conclusion drawn was that the oil movement is induced due to a moving space charge layer injected at the $\mathrm{HV}$ electrodes [3].

Takashima [4] using computer modelling determined the oil velocity in an oil filled vessel and developed the theory of a cylindrically shaped charge channel (see Fig. 1). This was later also observed by Atten [5]. The channel with radius $\alpha_{c}$ is extending from the tip of the electrode 1 to the plane electrode 2 and is created by the applied electric field E. Takashima has shown that $\alpha_{c}$ as well as the oil speed are constant over nearly the full length of the charge channel. His findings draw a correlation between the applied $\mathrm{HV}$, the oil velocity, and the radius of the oil channel.

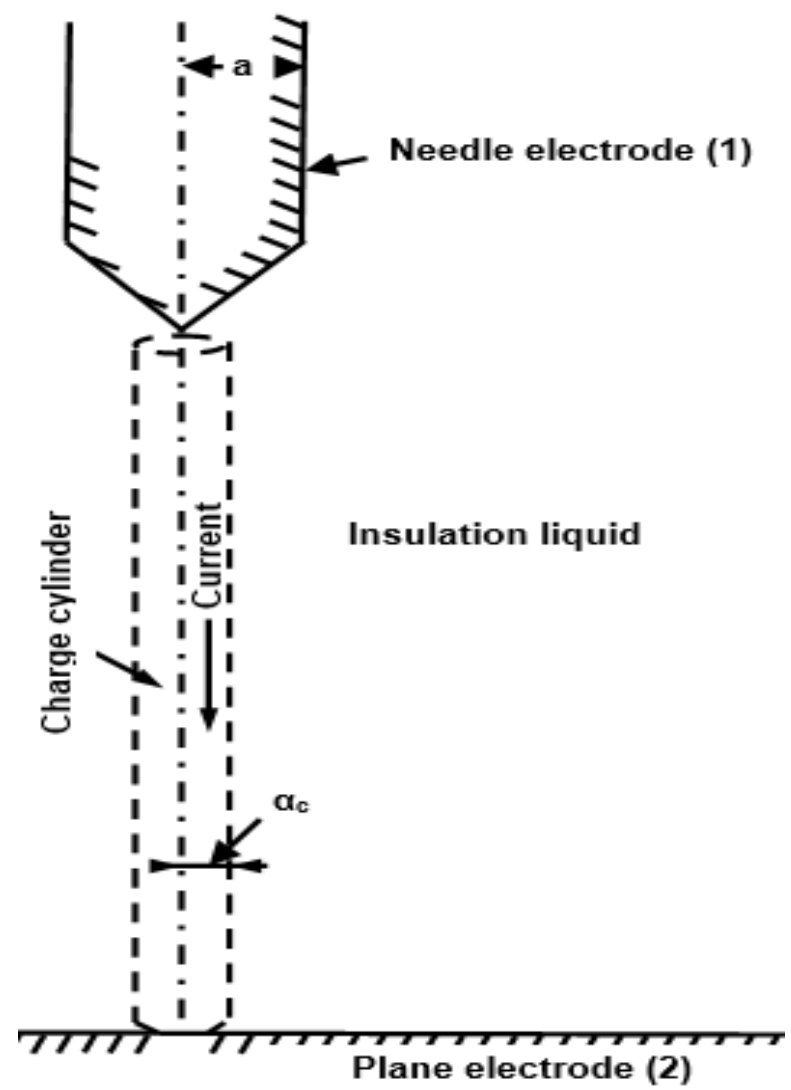

Figure 1: Charge channel generated in electrically stressed transformer oil. [4]

A bigger needle tip will also increase $\alpha_{c}$ while reducing the speed of oil movement in the charge channel [4]. 
As the result of the applied HV a current flow was observed this leads to energy dissipation into the oil and some of this energy will be transformed into heat $[4,5]$ subsequently warming the oil.

Such electric stress will lead to local breakdown in oil. This is known as partial discharge, PD [6]. A presence of the electric current due to discharge events is another factor leading to the localized oil heating [6].

By summarizing the above one can conclude the temperature distribution in oil can be represented by a thermal field resulted from events triggered by $\mathrm{HV}$ applied to electrodes.

We will show the effect of $\mathrm{HV}$ on the transformer oil temperature variations and changes in the oils index of refraction. By passing a laser beam between the electrodes through the charge channel. Analysing the changes of the laser beam properties we can visualize microscopic pre breakdown effects on the passing laser beam and predict an 'approaching' HV breakdown in oil.

\section{Experimental investigation of breakdown in oil transformer}

In order to exploit these events for $\mathrm{HV}$ breakdown prediction in transformer oil we have built an experimental setup shown in Fig. 2. The setup consists of a transparent cylindrical vessel (Fig. 2a) filled with transformer oil. Two electrodes (needle and flat) are fitted through the vessels walls. The gap between electrodes was set to $1.5 \mathrm{~cm}$. The needle tip radius was $1.5 \mathrm{~mm}$.

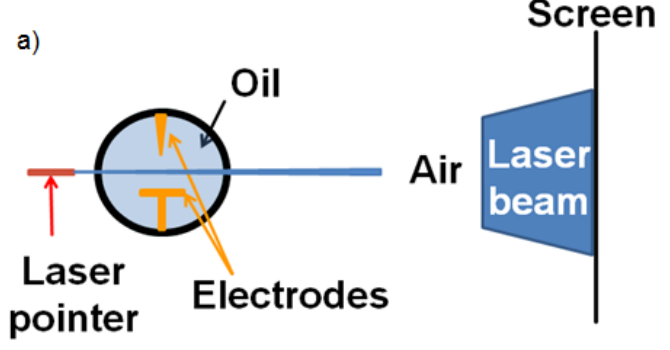

b)

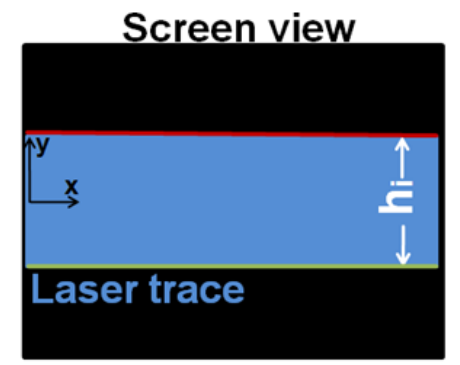

Figure 2: Measurement system setup a) top view of the setup; b) Screen to observe laser beam

A laser pointer was placed outside the vessel. The laser pointer was positioned $12.5 \mathrm{~cm}$ away from the midpoint of the oil vessel and adjusted to pass the laser beam through the oil between the vessels electrodes before hitting the screen (Fig. 2b). The total beam path length was $7.9 \mathrm{~m}$ (measured from the electrodes center point to the screen).
In each experiment the high voltage applied to the electrodes was raised in steps from $0 \mathrm{~V}$ to $8640 \mathrm{~V}, 12880 \mathrm{~V}$, $17520 \mathrm{~V}, 22200 \mathrm{~V}, 26680 \mathrm{~V}$, and $31470 \mathrm{~V}$ and kept constant for a minimum of 40 seconds.

The voltage was recorded in real time by a digital oscilloscope using the BenchVue software package. Beam shape changes on the screen were recorded by a camera.

From the recorded data we plotted changes of the laser beam width on the screen with any change of HV applied to electrodes. To record these beam width changes the Kinovea software package was used. The Software recorded the changing beam width shown in Fig. 4 by tracking the laser beam borders movement as shown in Fig. 3 as a function of time parallel to the applied HV across electrodes. The red (green) line respectively, represents the upper (lower) laser beam border the position is measured in pixels ( $\mathrm{px}, 1 \mathrm{px}=0.263 \mathrm{~mm}$ ) and is recorded on the lefty axis in Fig. 3.

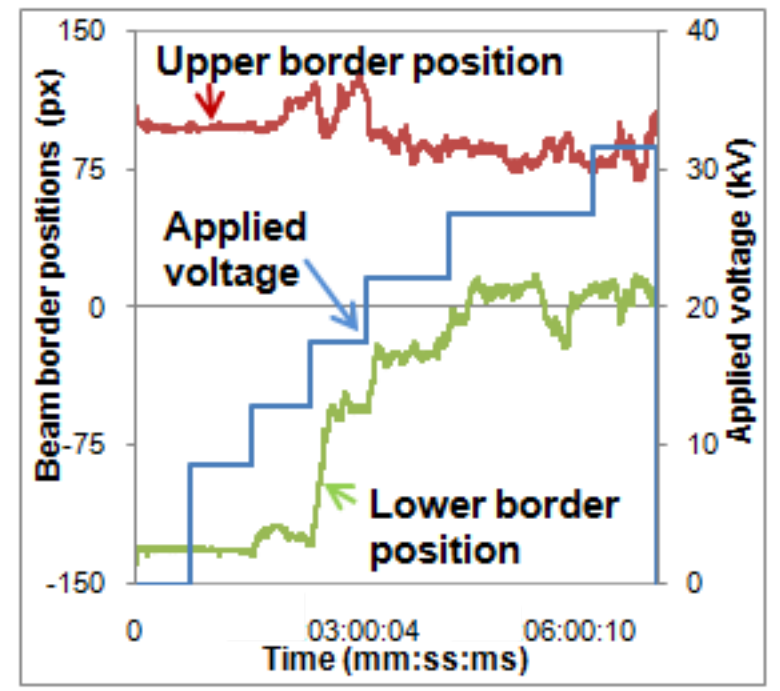

Figure 3: Change in the upper (red) and lover (green) laser beam borders position on the screen due to chances in applied voltage (blue)

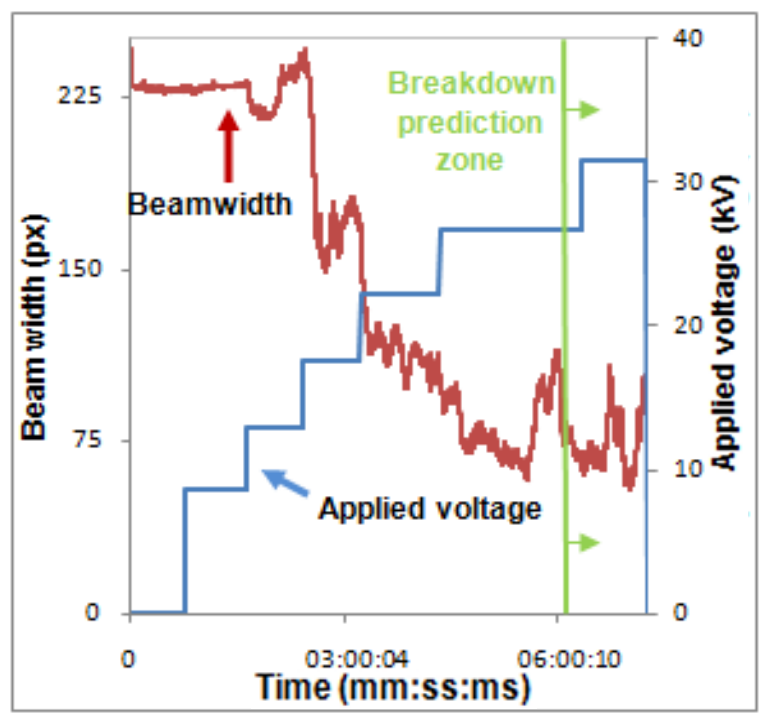

Figure 4: Changes in Laser beam width (red) due to chances in applied voltage (blue) 
The corresponding applied voltage in kilovolt $(\mathrm{kV})$ is the blue trace measured on the right y axis.

From the data analyses in Fig. 3 it can be said that both laser borders are stable until $12.9 \mathrm{kV}$. For higher voltage values both borders start to change positions but it is mainly the lower laser border that moves considerably as the $\mathrm{HV}$ increases which is a result of experimental arrangements. After the fifth voltage increase the lower border also stops moving but instead starts to oscillate and the insulation breakdown follows as it is clearly visible in Fig. 4. This behaviour was consistent in all our repeated experiments.

\section{Discussion}

The observed effects could be explained by taking into account the thermal stress in oil that is resulted from HV applied across the electrodes. The stress creates a dynamically changing cylindrical lens alongside the charge channel (see Fig. 5) and causes a thermal lensing effect in oil which in turn affects the passing laser beam. We applied a geometrical approach leading to a lens maker's formula for a thin lens [7].

$$
\frac{1}{f(V)}=\left(\frac{n_{\text {lens }}(V)-n_{\text {oil }}}{n_{\text {oil }}}\right) \frac{2}{R(V)}
$$

Where $f(V)$ is the focal length of this thermal cylindrical lens, $R(V)$ its radii of curvature and $n_{\text {lens }}(V)$ is the index of refraction represented by the refractive index of the warming up oil in the charge channel. It must be noted that all these three parameter are functions of the applied voltage $\mathrm{V}$. Were by $n_{\text {oil }}$ is the refractive index of the oil outside of the charge channel $\left(n_{\text {oil }}=1.47583\right.$ for $20^{\circ} \mathrm{C}$ [8]) and its value is constant. Any changes of the lens parameters via voltage $\mathrm{V}$ applied to the electrodes are directly responsible for shifts of the laser beam boundaries recorded and seen in Fig. 3 and Fig. 4. It can be easily shown that a change of the focal length $f$ will result in the change of the laser beam image width $h_{i}$ observed on the screen (see Fig. 2).

The 'creation' of this thermal lens is a direct result of changing properties of the oils index inside the charge cylinder by the applied voltage. The oil movement observed by Takashima and Atten $[4,5]$ is a contributing factor codifying the lens diameter. In first approximation the charge channel diameter $\alpha_{c}$ could be viewed identical with the lens parameter $R$. According to Takashima's observation the charge channel diameter $\alpha_{c}$ can increase up to 6 times with the increasing voltage [4]. Atten also observed the increase in the channel diameter with voltage [5]. This directly supports our proposal that increases in the charge channel diameter directly influence the lens radius $R$, thus its $f$ via Eq. 1 , and are results of changing HV.

We know that the refractive index of oil decreases with the temperature $\left(-3.75 \times 10^{-4} \mathrm{dn} / \mathrm{dT}^{\circ} \mathrm{C}^{-1}\right)$ [8]. Any increases in $\mathrm{HV}$ lead to higher currents [3] in the charge channel and will cause oil heating in the channel thus leading to voltage dependent changes of the refractive index inside the channel. This heat field takes the shape of the moving oil and is directly responsible for creation of the described cylindrically shaped lens like an object alongside the channel. Stishkov simulated and afterwards observed the geometry of the moving oil [9] and came to similar results as Takashima. The lens refraction index $\mathrm{n}_{\text {lens }}(\mathrm{V})=\mathrm{n}_{\text {hot-oil }}(\mathrm{V})$ closely follows oil temperature changes within the charge channel, from the hottest value

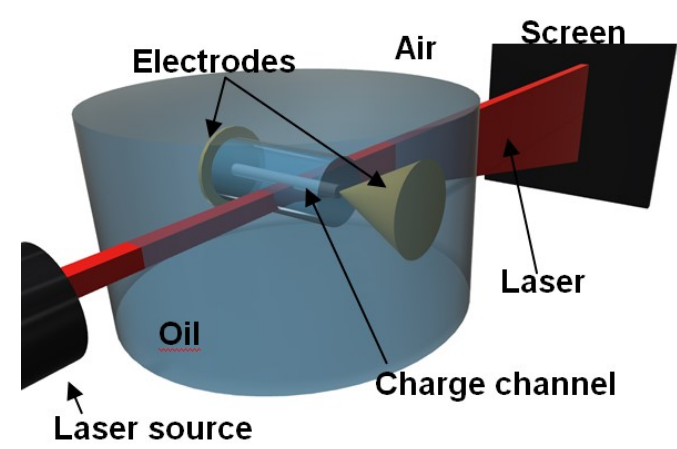

Figure 5: Laser beam affected by a thermal gradient lens created by a charge channel in HV stressed insulation between electrodes

(lowest index of refraction) in its middle to lower temperatures (higher indexes) in outward directions according to the oil speed contours, until the temperature equilibrium with the oil surrounding the charge channel is reached. Because these are HV related changes of the lens parameters they are directly responsible for the observed beam movements seen on the screen and based on our measurements quantified in Fig. 3 and Fig. 4.

When it comes to HV breakdown prediction, just before this event, partial discharges of high energy lead to the rapid development of gas channels between the electrodes [6]. These create shockwaves [6] and lead to the disruption of the laminar flow between the electrodes $[4,9]$ within the charge channel (the host of the discussed thermal cylindrical lens). We expect that these events are disrupting the uniformity of this lens strongly affecting the passing laser beam. This manifest itself as violent laser beam movements observed on the screen just before the breakdown point as recorded in Fig. 4. The movement is mainly due to the change in the lens geometry and local changes in $n_{\text {lens }}$ due to the additional heating from the PD event. The lens then recovers to its initial geometry due to the moving oil closing the gas channels and carrying away or dissolving the additional heat. By observing the laser beam on the screen we were able to predict the point of total insulation breakdown.

\section{Conclusion}

We described a measurement system which uses a simple optical concept for monitoring microscopic prebreakdown events in liquid insulators such as oil. The system has been built and demonstrated in the laboratory and is capable of predicting high voltage breakdowns. A simple theory based on a thermal lens build up between electrodes within the charge channel as a result of HV is presented to explain the system operation and observed measurement results. 


\section{References}

[1] M. Judd, L. Yang, I. Hunter, "Partial Discharge Monitoring for Power Transformers Using UHF Sensors Part 1: Sensors and Signal Interpretation," IEEE Electrical Insulation Magazine, Vol. 21, No.2; March/April 2005

[2] J. Cross, M. Rakano, S. Savannis, "Electric Stress Induced motion in Transformer Oils under $60 \mathrm{~Hz}$ Stress, "Journal of Electrostatics, 7 page 361--372 361; Elsevier Scientific Publishing Company, Amsterdam; 1979

[3] P. Atten, M. Haidara, "Electrical Conduction and EHD Motion of Dielectric Liquids in a Knife-plane electrode Assembly," IEEE Transactions on Electrical Insulation Vol. EI20 No.2, April 1965

[4] T. Takashima, "I-V Characteristics and Liquid Motion in Needle-to-Plane and Razor Blade- to- Plane Configurations in Transformer Oil and Liquid Nitrogen," IEEE Transactions on Electrical Insulation Vol. 23 No. 4, August 1988, page 645-658
[5] P. Atten, B. Malraison, M. Zahn, "Electro hydrodynamic Plumes in Point- plane Geometry," IEEE Transactions on Dielectrics and Electrical Insulation, Vol. 4 No. 6, 1997

[6] M. Butcher at al, "Conduction and Breakdown Mechanisms in Transformer Oil"; IEEE Transactions on plasma science, Vol. 3, No 2; April 2006

[7] J. Greivenkamp, "Field Guide to Geometrical Optics," SPIE Press Field Guides Vol FG01; Washington, USA; 2004

[8] L. Watters, "Liquid Refractive Index - Mineral Oil," Department of commerce USA, National Institute of Standards \&Technology, Certificate, Standard Reference Material ${ }^{\circledR} 1922$ [9] Yu.K. Stishkov, V.A. Chirkov, "Features of electro hydrodynamic flows in needle- plane electrode system," IEEE International Conference, Dielectric Liquids, 2008. ICDL 2008 